\title{
Nutritional studies of rice bran oil
}

\begin{abstract}
Rice bran oil (RBO) is not a popular oil worldwide, but its demand is increasing due to its popularity as a "healthy oil" in Asian countries, particularly in Japan, Korea, India, China, and Indonesia. RBO is an excellent cooking oil and salad oil due to its high smoke point and delicate flavor. It is unique among edible oils due to its rich source of commercially and nutritionally important phytoceuticals such as oryzanol, lecithin, squalene, phytosterols, polyphenols, tocopherols, tocotrienols, and many more. Many studies on humans and animals have shown that RBO is as effective as some other vegetable oils that are rich in polyunsaturated fatty acids in lowering plasma cholesterol levels and lipoprotein profiles. In some cases, RBO was found to lower plasma cholesterol more effectively, and this effect can be attributed to the occurrence of these other specific components in RBO. This chapter provides a comprehensive review of the phytonutrients from RBO that have shown promising disease-preventing and health-related benefits in experimental research studies.
\end{abstract}

Keyword: $\gamma$-Oryzanol; Hypocholesterolemic; Hypolipidemia; Anticancer; Antidiabetic; Phytosterols; Squalene; Tocotrienols; Tocopherols; Antiinflammatory; Antitumor; Cardioprotective 